\title{
The effects of vitamin D supplementation on interictal serum levels of calcitonin gene-related peptide (CGRP) in episodic migraine patients: post hoc analysis of a randomized double-blind placebo- controlled trial
}

Zeinab Ghorbani ${ }^{1}$, Pegah Rafiee ${ }^{2}$, Akbar Fotouhi ${ }^{3}$, Samane Haghighi ${ }^{4}$, Reyhaneh Rasekh Magham ${ }^{5}$, Zeynab Sadat Ahmadi ${ }^{6}$, Mahmoud Djalali ${ }^{1}$, Mahnaz Zareei ${ }^{1}$, Soodeh Razeghi Jahromi ${ }^{7}$, Sahar Shahemi ${ }^{7,8}$, Maryam Mahmoudi ${ }^{1,9,10^{*}}$ and Mansoureh Togha $a^{4,11^{*}}$ (D)

\begin{abstract}
Background: Emerging evidence showed promising effects of vitamin D on headaches characteristics. Thus, it seems there is still a need for more researches to clarify the mechanisms by which this vitamin exerts anti-migraine effects.

Methods: The present study was conducted as a 16-week randomized double-blind placebo-controlled trial on 80 episodic migraine patients allocated in 2 parallel groups each consisted of 40 patients who received vitamin D $2000 \mathrm{IU} / \mathrm{d}$ or placebo. At baseline and after the intervention completion, headache diaries and migraine disability assessment questionnaire (MIDAS) were used to assess migraine related variables in patients. Also, interictal serum concentration of calcitonin gene-related peptide (CGRP) (as the dominant mediator of migraine pain pathogenesis) was evaluated using ELISA method.

Results: The mean (SD) of age in the vitamin D and placebo groups was 37 (8) and 38 (12) years, respectively. ANCOVA test adjusted for baseline values, and confounders showed vitamin D supplementation resulted in a significant improvement in MIDAS score after 12 weeks in the intervention group (21.49 (16.22-26.77)) compared to placebo (31.16 (25.51-36.82) $P$ value: 0.016). Moreover, after controlling for baseline levels, and other variables using ANCOVA, CGRP level was appeared to be significantly lower following vitamin D supplementation (153.26 (133.03-173.49) $\mathrm{ng} / \mathrm{L})$ than the patients in the placebo arm (188.35 (167.15-209.54) ng/L) $(P$ value $=0.022)$.

(Continued on next page)
\end{abstract}

\footnotetext{
*Correspondence: maryam.mahmoudi73@gmail.com; togha1961@gmail.com

1 Department of Cellular and Molecular Nutrition, School of Nutritional

Sciences and Dietetics, Tehran University of Medical Sciences, Tehran, Iran

${ }^{4}$ Headache Department, Iranian Center of Neurological Research,

Neuroscience Institute, Tehran University of Medical Sciences, Tehran, Iran

Full list of author information is available at the end of the article
}

(c) The Author(s). 2020 Open Access This article is distributed under the terms of the Creative Commons Attribution 4.0 International License (http://creativecommons.org/licenses/by/4.0/), which permits unrestricted use, distribution, and reproduction in any medium, provided you give appropriate credit to the original author(s) and the source, provide a link to the Creative Commons license, and indicate if changes were made. 


\begin{abstract}
(Continued from previous page)
Conclusion: According to the current findings, vitamin D supplementation in episodic migraineurs, particularly in those with migraine with aura, may potentially improve migraine headache characteristics and disability probably through attenuating CGRP levels. Therefore, these results could provide a new insight into anti-nociceptive effects of vitamin D; however, more studies are required to confirm our findings.
\end{abstract}

Trial registration: The trial is registered in the Iranian registry of clinical trials (IRCT) at 11 July 2018, with IRCT code: IRCT20151128025267N6.

Keywords: Cholecalciferol, Disability, Headache, CGRP, Migraine with Aura

\section{Background}

According to the report of global burden of disease (GBD) 2016, migraine headache has been established as the leading disability cause in the globe among under 50 years old [1]. It has been estimated that migraine affects about $11 \%$ of adults with a major impact on those aged between 25 to 55 years $[1,2]$, which consequently lead to the high headache burden on individuals, communities, health care systems, and societies [3]. Migraine is 2-3 times more common in women than in men. Migraine attacks are usually more prolonged, with higher intensity and more disability among females [4].

Two main categories of migraine include episodic migraine (having $<15$ headache days per month) and chronic migraine (having $\geq 15$ headache days per month, of which at least 8 days are with migraine features or response to Triptans, for at least 3 months) [5]. If patients who suffer from episodic migraine, especially those with frequent attacks, are not treated appropriately, it can lead to chronic type which is reported to be more severe with higher disability $[6,7]$.

Both genetics and environment are supposed to be related to migraine development. Some dietary factors, misuse of caffeine, hormonal fluctuations, high stress level, increased weight, smoking, and suffering from tension type headache or medication overuse headache are among the suggested factors affecting migraine susceptibility and or chronification [8].

There is no established mechanism to explain migraine pathogenesis, although there have been much efforts directed in this field. The current evidence suggest a variety of mechanisms which are thought to be involved in pathophysiology of migraine including cortical spreading depression (CSD), trigeminovascular pathway activation (a pathway for nociceptive information conduction), neuro-inflammation as well as dysfunction in vascular system [9]. Also, different neuropeptides such as calcitonin gene-related peptide (CGRP), and pituitary adenylate cyclase-activating peptide (PACAP) with vasoactive properties are believed to be released during migraine attacks. The release of these factors could affect activation of trigeminovascular system and subsequently might induce mast cells degranulation, vasodilatation of arteries, and plasma leakage, which all are supposed to be involved in migraine attacks initiation [9].

Current evidence highlighted a need for compounds that suppress the neuro-inflammatory state in migraine as novel effective medications in its treatment $[8,10$, 11]. Keeping this in mind, adverse effects that related to the present migraine pharmacological treatments (including weight reduction or weight gain, fatigue/ sleepiness, sweeting, night dreams, gastrointestinal upset, hypotension or reduced concentration) might negatively impact on the patients' adherence to drug therapy, and therefore reduce the efficacy of treatment procedure [12-15]. Hence, applying novel therapeutic approaches including nutritional agents could be promising strategies that can favorably modify migraine characteristics and its associated disability [8]. Magnesium, omega 3, melatonin, coenzymes Q10, riboflavin and folate are among the most studied dietary supplements that have been suggested to be beneficial in prophylaxis of migraine $[16,17]$. Also, vitamin $\mathrm{D}$ is one of the dietary agents that has been taken into consideration for pain and migraine improvement [18-23]. In this regard, our team has recently published a review paper aiming to address the association between vitamin $\mathrm{D}$ and headache [8]. It was estimated that about $45-100 \%$ of migraine or headache patients might suffer from deficiency/insufficiency of vitamin D. It was further shown that there seems to be a negative correlation between serum vitamin D concentrations and headaches frequency [8]. Also, in our previous paper [24], we showed that supplementation with vitamin D (2000 IU/d) could reduce number of headache attacks, decrease the need for analgesic consumption and attenuate intensity and duration of headaches. We also observed that this vitamin might suppress neuro-inflammation in migraineurs through lowering the levels of inducible nitric oxide synthase (iNOS) and probably interleukin (IL)-6 [24]. However, it seems there is still a need to further clarify the mechanisms by which vitamin $\mathrm{D}$ exerts anti-migraine effects. Therefore, in the same study, we aimed to explore changes in interictal levels of the dominant mediator of migraine pain pathogenesis [25] (serum CGRP) following prophylactic administration of vitamin D. In addition, 
vitamin $\mathrm{D}$ effects on migraine associated disability was aimed to be determined.

\section{Methods}

\section{Study design}

The details on study design and data collection has been described in our previous publication [24]. Briefly, the present study was conducted as a 16-week (12-week intervention following a 4-week baseline period) randomized double-blind placebo-controlled trial on 80 episodic migraineurs aged between 18 and 45 years to investigate the effects of $2000 \mathrm{IU} / \mathrm{d}$ vitamin D supplementation on headache characteristics and CGRP levels compared with placebo.

\section{Participants and settings}

The current randomized clinical trial (RCT) was performed in Tehran, Iran from July 2018 to July 2019 . Totally, $98 \mathrm{mi}-$ graine patients who were referred to the tertiary headache clinic of Sina University Hospital, were interviewed for the current trial. Inclusion criteria were diagnosis of episodic migraine (EM) according to headache characteristics and the International Classification of Headache Disorders, 3rd edition (ICHDIII criteria) [5], age between 18 to 45 years, having body mass index (BMI) between $18.5-30 \mathrm{~kg} / \mathrm{m} 2$ and suffering from migraine for at least 6 months prior to study. Exclusion criteria were unwillingness to participate in the study, suffering from medication overuse headache in 3 months prior to study, taking vitamin D supplements in 3 months prior to study, taking magnesium, calcium, zinc, vitamin $\mathrm{B}$ groups or vitamin $\mathrm{C}$ supplements during the study period, taking anti-epileptic drugs such as topiramate, sodium valproate and carbamazepine, taking thiazide diuretics, glucocorticoid, statins or orlistat, taking antipsychotic drugs. Also, menopause, pregnancy and lactation, and suffering from gastrointestinal disorders, liver and kidney disorders, cancer, sarcoidosis, rickets, and osteomalacia according to physician diagnosis and/or past medical history were among the other exclusion criteria.

\section{Participants' randomization and blinding}

After initial examination by the study expert headachespecialist neurologists, 32 subjects were excluded due to exclusion criteria as illustrated in Fig. 1. From the beginning

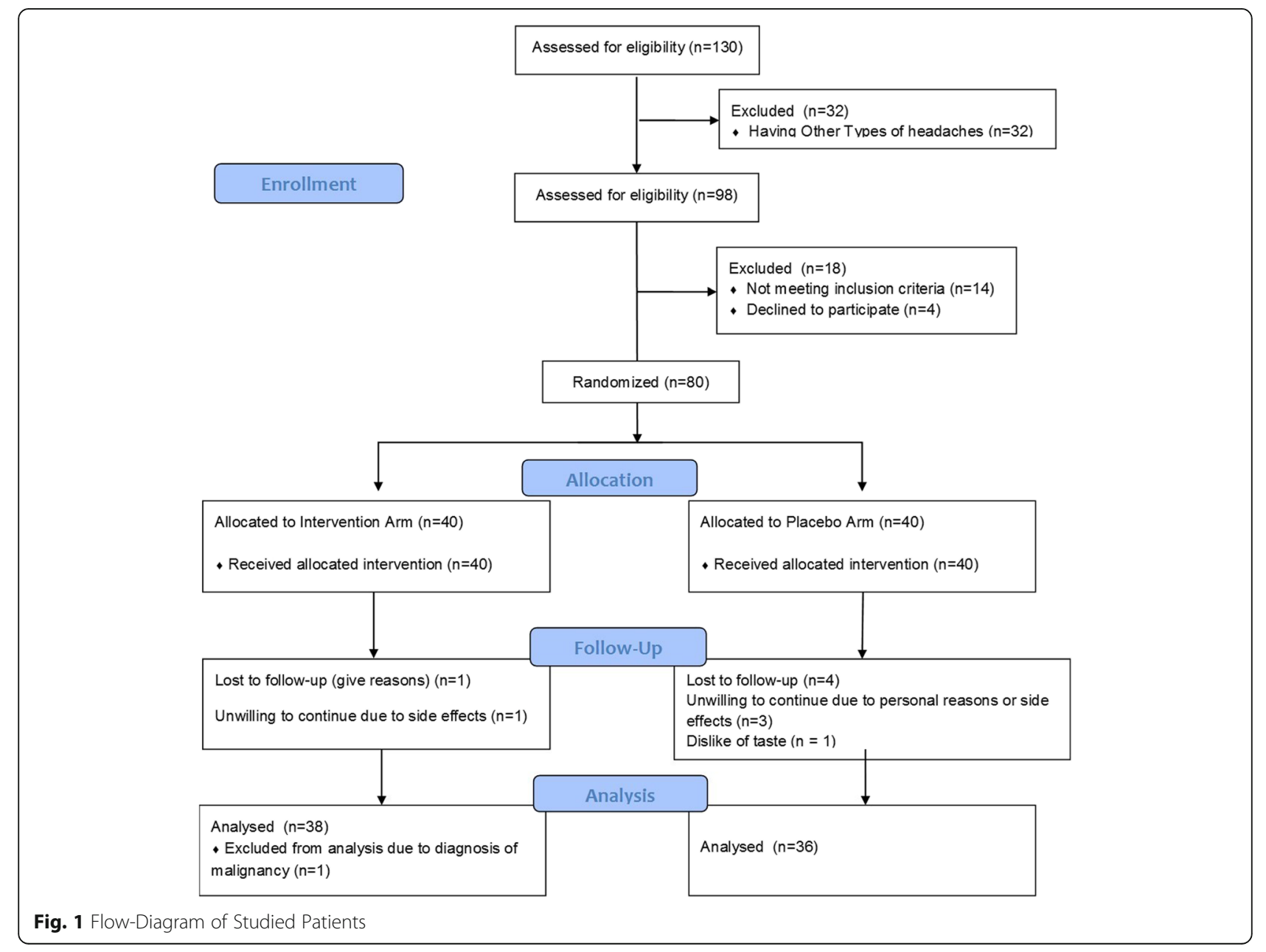


of the study, A (vitamin D supplement) and B (placebo) codes were available to the researchers to recruit the patients. Only one of the staff of headache department was aware of codes. Block randomization was applied to ensure that both participants and researchers were blind to treatment allocation. Thus, 80 patients who fulfilled the inclusion criteria were randomly allocated in a 1:1 ratio in blocks of 4 to receive either A or B supplements and stratified based on gender. Since assignment of patients was concealed until statistical analysis completion, the participants, neurologists, and investigators were blinded to the group allocation throughout the trial.

\section{Data collection}

At the first visit, all demographic and socioeconomic information including age, sex, marital status, educational status, and job, headache onset years, having migraine with/without aura, familial history of migraine and smoking status, number and type of medications, and data on past medical history of patients were collected through face to face interview by an expert researcher.

Anthropometric data including weight, and height were also evaluated at study entry and the end of the trial as follow: body weight was assessed to the nearest $0.5 \mathrm{~kg}$ and height to the nearest $0.1 \mathrm{~cm}$ applying a Seca 755 dial column medical scale and standard stadiometer, respectively. BMI was calculated as weight in kilograms divided by height in square meters.

\section{Intervention}

Patients in the intervention group received 1 pearl containing $2000 \mathrm{IU}$ of vitamin $\mathrm{D} / \mathrm{d}$ in addition to routine medications prescribed by our study neurologists. The patients in the placebo group were given 1 placebo pearl which was indistinguishable from vitamin $\mathrm{D}$ pearls in taste and appearance. Throughout the trial, the subjects were allowed to consume their usual acute/ prophylactic drugs, though they were asked not to change their medications during the trial. Both vitamin $\mathrm{D}$ and placebo pearls were produced by Zahravi Pharmaceutical Company, Tabriz, Iran.

\section{Outcome measures}

\section{Headache diaries assessment}

All participants received a 30-days headache diary to fill out the migraine characteristics (designed by the senior researcher, Prf. M.T and has been described in details previously) [26]. Briefly, the participants were asked to complete their headache diaries during a month prior to beginning the intervention and also the last month of trial. Detailed results of present RCT on headache characteristics based on these diaries have been reported previously [24]. To better understand current findings, we only mentioned the results on the number of headache days per month.

In addition, at the end of investigation we asked the participants to roughly rate their overall perception of headaches improvement following the current intervention using a six-point Likert scale, ranging from 1 (less than $10 \%$ ) to 6 (more than $70 \%$ ) [27].

\section{Migraine disability assessment questionnaire (MIDAS)}

At baseline and after the intervention completion, MIDAS questionnaire was used to assess migraine related disability in patients. The questionnaire includes seven questions that examine the levels of impact of headaches on work, home, social and leisure activities over the past 3 months. Questions 1 through 5 of this questionnaire assess the migraine-related decline in performance. The questionnaire was validated previously in Iran [28].

\section{Blood collection and serum analysis}

At the first visit and the end of the trial, $5 \mathrm{ml}$ blood samples were obtained from all the patients to assess serum concentration of 25OHD and CGRP. Blood samples were centrifuged at $4{ }^{\circ} \mathrm{C}$ for $10 \mathrm{~min}$ and serum samples were kept at $-80^{\circ} \mathrm{C}$ until laboratory analysis. Vitamin $\mathrm{D}$ levels in the form of serum 25OHD (S25OHD) were evaluated using chemiluminescence immunoassays (CLIA) method. Serum level of CGRP was evaluated using enzyme linked immunosorbent assay (ELIZA) method and Crystal Day kit, E1061 Hu, 96 tests (Crystal day, China). The interval of time from the last attack of headache to the day of blood samples collection was also recorded.

\section{Compliance, adverse events and patients' satisfaction score}

Compliance was indicated using tablet count after 12week of intervention. A studied participant was considered compliant if $\geq 70 \%$ of the tablets had been used throughout the trial. During study follow-up, the compliance was additionally stimulated by weekly telephone calls and reminding the participants to use the supplements. Also, after 12week trial, S25OHD status was evaluated in order to assess the compliance of the subjects in the intervention arm. Also, patients were asked to report any minor or major adverse effects which were likely to be associated with supplementation. The subjects were also asked to rate their satisfaction with participation in the study using a five-point Likert scale ranging from 1 (very dissatisfied) to 5 (very satisfied) [27].

\section{Sample size calculation and statistical analysis}

According to the below formula, it was assumed that by estimating 40 samples in each group with an average of 8 headache day/month (standard deviation (SD): 4 days), 
aiming to detect at least 4 days reduction in headache days, $90 \%$ statistical power will be achieved $(\alpha=0.05$, $\beta=0.90$, and $S=4$ and $d=4$ ) [19].

$$
n=2 \frac{\left(Z 1-\frac{\alpha}{2}+Z 1-\beta\right)^{2} S_{2}}{(d)^{2}}
$$

Means (SD) were used to describe the quantitative variables and frequencies (percentage, \%) were reported for categorical variables. Within and between groups comparisons were made using paired sample t-test or either independent $\mathrm{t}$ test or Chi square test, respectively. Further, one-way analysis of variance (ANOVA) was used to compare between groups when aiming to assess variables changes before and after supplementation with vitamin D or placebo in episodic migraine patients with/without aura. In order to analysis the effects of intervention on headache characteristics, MIDAS score, and serum CGRP levels, analysis of covariance (ANCOVA) adjusted for baseline values and confounders was applied. In the case of using ANCOVA, the adjusted means and 95\% confidence intervals (95\% CI) were also reported. Pearson correlation test was used to determine the correlation between changes in S25OHD and outcome variables. The figures was prepared by GraphPad Prism 5.0 (GraphPad Software, Inc.). Significance level was defined as $P<0.05$ level. All statistical analyses were performed using SPSS program version 19 (IBM Corporation, New York, USA).

\section{Results}

\section{Baseline, clinical and anthropometric characteristics}

Figure 1 displays the studied subjects flow-diagram. Initially, 130 headache patients were examined by our study headache-specialists of whom 32 had other types of headache concurrent with migraine including cervicogenic headache, or secondary headaches. Thus 98 migraineurs were assessed for eligibility criteria of whom 80 were included in the trial; and randomized into vitamin D $(n=40$, $80.0 \%$ women) or placebo group ( $n=40,80.0 \%$ women). Four subjects in the placebo group and 2 patients in the intervention arm were lost to follow-up. The reasons are described in Fig. 1.

Table 1 displays the baseline characteristics, and clinical and anthropometric data of the studied participants. The two studied groups did not differ significantly in age, sex, headache onset years, socio-economic status, having migraine with/without aura, familial history of migraine or smoking status. The mean (SD) of age in the vitamin D and placebo groups was 37 (8) and 38 (12) years, respectively. On average, headache onset years in the patients in the intervention and control group were about 12 and 11 years, respectively. Additionally, approximately $32.5 \%$ of patients who received vitamin D supplements and $25.0 \%$ of the placebo taking patients were suffering from migraine with aura. The mean BMI was approximately around $25.83 \mathrm{~kg} / \mathrm{m} 2$ in vitamin D group, and $25.36 \mathrm{~kg} / \mathrm{m} 2$ in placebo group before and after the study, with no significant differences between groups.

\section{Serum vitamin D status}

Mean baseline S25OHD levels among patients received placebo was significantly higher than those in the vitamin D group $(P$ value $=0.017)$; however, after 12 weeks of study, serum levels of this vitamin significantly increased in the vitamin D group (from 27.31 to $39.65 \mathrm{ng}$ / $\mathrm{ml} ; P$ value $<0.001)$ compared to no significant changes observed in the placebo group (from 33.70 to $33.95 \mathrm{ng}$ / $\mathrm{ml})(P$ value for between group comparison $=0.062)$.

\section{Medications consumption}

The medications consumption of both studied groups at baseline and after the intervention consisted of abortive and prophylactic drugs are demonstrated in Fig. 2. There was not any significant differences between groups before and after the trial on type of abortive/prophylactic medications use, except for using triptans in the placebo group which was significantly higher than that of vitamin D group after the trial $(P=0.024)$.

\section{The number of headache days and frequency of attacks}

Detailed results of present RCT on headache characteristics have been reported previously [24]. Patients in treatment and placebo group were estimated to experience about 8.04 and 7.69 headache days per month, respectively. No significant differences were noted regarding baseline values of number of headache days between the groups. Paired t-test revealed statistically significant reduction in the number of headache days among the patients who received vitamin $D$ supplements (mean changes: -2.90 (3.03); $P$ value $<0.001$ ), whereas there were no significant changes observed within the placebo group when comparing to the baseline data. Following the trial, ANCOVA test adjusted for baseline values and confounders demonstrated that patients in vitamin $\mathrm{D}$ group had significantly lower headache days (4.71 day/ month) in comparison with placebo group (6.43 day/ month) ( $P$ value from ANCOVA test $=0.031)$.

\section{Migraine disability}

There were no significant differences in baseline MIDAS scores in vitamin D and placebo groups (mean values = 29.75 and 36.87, respectively). Vitamin D supplementation resulted in a significant improvement in MIDAS score after 12 weeks in the intervention group (21.49 (16.2226.77)) compared to placebo (31.16 (25.51-36.82) $P$ value from ANCOVA test $=0.016)$ ). Within group comparisons revealed this score significantly reduced in vitamin $D$ group (mean changes: -10.38 (12.29); $P$ value $<0.001$ ), 
Table 1 Baseline demographic, anthropometric and clinical data of episodic migraine patients in a randomized controlled trial of vitamin D vs. placebo

\begin{tabular}{|c|c|c|c|}
\hline & Vitamin D $(n=40)$ & Placebo $(n=40)$ & $P$ value \\
\hline Age (year) & $37(8)$ & $38(12)$ & 0.819 \\
\hline Headache Onset years (year) & $12(8)$ & $11(8)$ & 0.679 \\
\hline Body mass index (kg/m2) & $25.83(4.07)$ & $25.36(4.29)$ & 0.613 \\
\hline \multicolumn{4}{|l|}{ Sex } \\
\hline Female & $32(80.0 \%)$ & $32(80.0 \%)$ & \multirow[t]{2}{*}{1.000} \\
\hline Male & $8(20.0 \%)$ & $8(20.0 \%)$ & \\
\hline \multicolumn{4}{|l|}{ Marital Status } \\
\hline Married & $31(77.5 \%)$ & $26(65.0 \%)$ & \multirow[t]{3}{*}{0.339} \\
\hline Single & $9(22.5 \%)$ & $13(32.5 \%)$ & \\
\hline Widow/widower & $0(0 \%)$ & $1(2.5 \%)$ & \\
\hline \multicolumn{4}{|l|}{ Educational Status } \\
\hline High school & $13(32.5 \%)$ & $16(40.0 \%)$ & \multirow[t]{4}{*}{0.293} \\
\hline $\mathrm{BSC}$ & $11(27.5 \%)$ & $7(17.5 \%)$ & \\
\hline MS & $8(20.0 \%)$ & $13(32.5 \%)$ & \\
\hline $\mathrm{PhD}$ and higher & $8(20.0 \%)$ & $4(10.0 \%)$ & \\
\hline \multicolumn{4}{|l|}{ Job } \\
\hline No job/house wife & $12(30.0 \%)$ & $16(40.0 \%)$ & \multirow[t]{4}{*}{0.480} \\
\hline Student & $1(2.5 \%)$ & $3(7.5 \%)$ & \\
\hline Employee & $23(57.5 \%)$ & $17(42.5 \%)$ & \\
\hline Self employed & $4(10.0 \%)$ & $4(10.0 \%)$ & \\
\hline \multicolumn{4}{|l|}{ Migraine Aura } \\
\hline Migraine with aura & $13(32.5 \%)$ & $10(25.0 \%)$ & \multirow[t]{2}{*}{0.459} \\
\hline Migraine without aura & $27(67.5 \%)$ & $30(75.0 \%)$ & \\
\hline \multicolumn{4}{|l|}{ Familial History of Migraine } \\
\hline Yes & $26(65.0 \%)$ & $32(80.0 \%)$ & \multirow[t]{2}{*}{0.133} \\
\hline No & $14(35.0 \%)$ & $8(20.0 \%)$ & \\
\hline \multicolumn{4}{|l|}{ Smoking Status } \\
\hline No & $37(92.5 \%)$ & $35(87.5 \%)$ & \multirow[t]{4}{*}{0.549} \\
\hline Yes & $0(0 \%)$ & $1(2.5 \%)$ & \\
\hline More than 5 cigarette per week & $3(7.5 \%)$ & $4(10.0 \%)$ & \\
\hline Less than 5 cigarette per week & $0(.0 \%)$ & $0(0 \%)$ & \\
\hline
\end{tabular}

Values are expressed as mean (SD) or number (\%) as appropriate

\#Using either Independent $\mathrm{t}$ test or Chi square according to the type of data

while there were no significant changes noted within the placebo group (Table 2).

\section{Comparison between episodic migraine patients with or without aura}

Following 12 weeks of intervention, number of headache days and disability score significantly decreased in vitamin $\mathrm{D}$ treated migraine patients either those with aura or without aura $(P$ value $\leq 0.002)$. Interestingly, migraineurs with aura who received vitamin $D$ supplements had the highest reduction of mean frequency of headache days per month $(-4.58)$ and MIDAS score $(-16.92)$ as compared to other studied groups ( $P$ value $\leq 0.001)$ (Table 3$)$. 


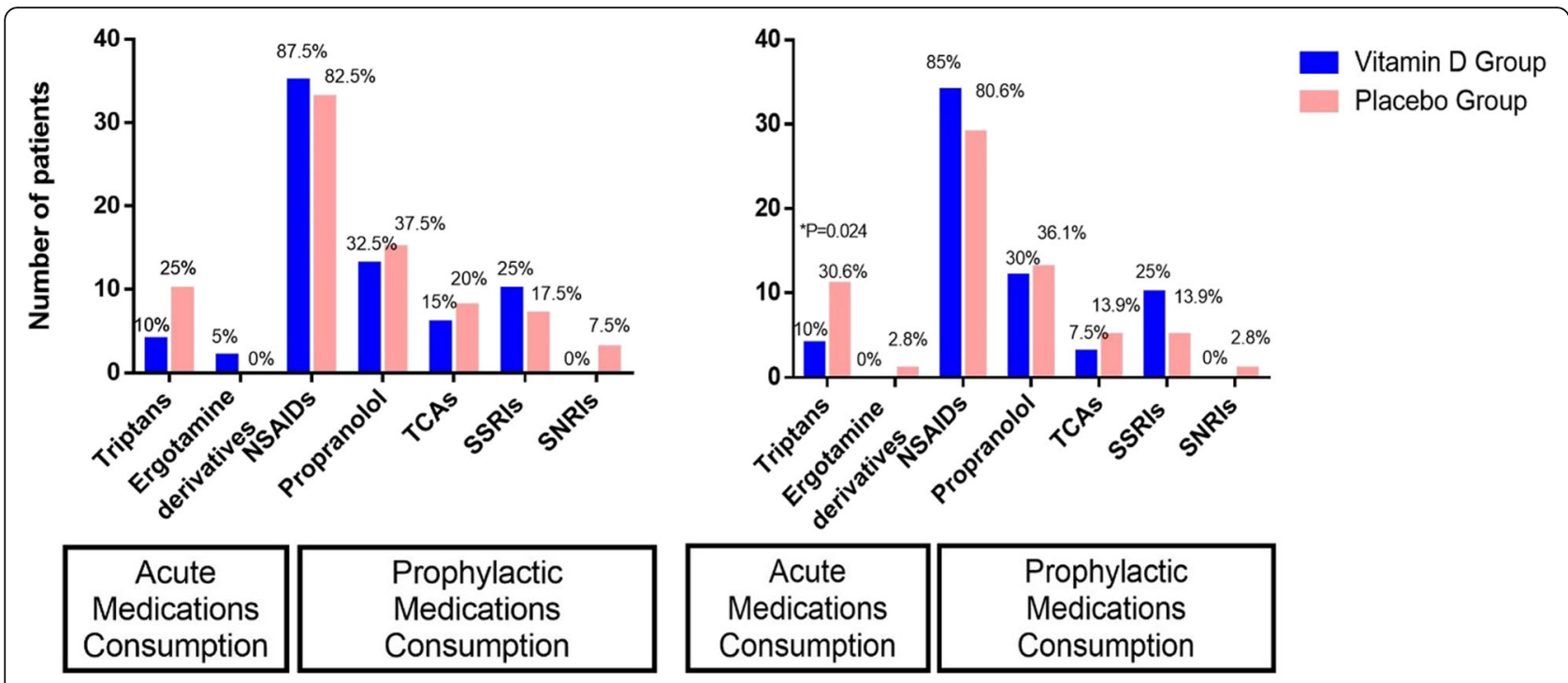

Drug Treatment Before

\section{Drug Treatment After}

Fig. 2 Types of Medications Used By Studied Patients Throughout The Trial. NSAIDs, Nonsteroidal anti-inflammatory drugs. TCAs, Tricyclic antidepressants. SSRIs, Selective serotonin reuptake inhibitors. SNRIs, Serotonin-Norepinephrine Reuptake inhibitors

Patients' overall perception of headaches improvement About $17.5 \%(n=7)$ and $10 \%(n=4)$ of patients in the vitamin D group reported $50-70 \%$ and $>70 \%$ overall improvement in their headaches, respectively; while majority of patients in control group reported $<20 \%$ improvement $(P$ value $<0.001)$ (Fig. 3$)$.

\section{Serum analysis of CGRP levels}

At baseline, mean time from the last attack to the day of blood samples collection was reported to be about 4 days in both vitamin $\mathrm{D}$ and placebo groups. At the end of trial, mean days since the last attack was estimated as 5 days in vitamin D group and 4 days in placebo group. The changes in serum concentrations of CGRP in two studied groups before and after supplementation with vitamin $\mathrm{D}$ or placebo are demonstrated in Table 2. At baseline, patients in vitamin $\mathrm{D}$ group had a slightly higher serum levels of CGRP (mean values $=195.27$ and166.05 ng/L, respectively; $P$ value $=0.063$ ). Within group comparisons showed CGRP levels significantly reduced in the vitamin $\mathrm{D}$ group compare to baseline (mean changes: -27.31 (59.69); $P$ value $=0.006$ ), while it slightly increased in the placebo group (mean changes: $12.57 \pm 23.85) ; P$ value $=0.004)$. After the trial, the results of independent $t$ test showed no significant differences in mean CGRP concentration of the two studied group; however, after controlling for baseline CGRP levels, age, sex, BMI change, the years having headache, and time from last attack using ANCOVA, CGRP level was appeared to be significantly lower following vitamin $D$ supplementation among patients in the intervention group (153.26 (133.03-173.49) ng/L) than the patients in the placebo arm (188.35 (167.15-209.54) ng/L ( $P$ value: $0.022)$ ).

\section{Correlation between changes in S25OHD and headache related variable}

Figure 4 illustrates the Pearson correlation analysis between S25OHD changes and changes in the number of headache days (Fig. 4a), changes in migraine disability score (Fig. 4b) CGRP levels changes (Fig. 4c) over the 3month supplementation with $2000 \mathrm{IU} / \mathrm{d}$ vitamin D or placebo. Non-significant negative correlations were detected between changes in S25OHD and changes in number of headache days per month $(r=-0.201, P$ value $=0.094)$, MIDAS score $(r=-0.176, P$ value $=$ $0.172)$, and CGRP levels $(r=-0.144, P$ value $=0.219)$ throughout the intervention (Fig. 4).

\section{Adverse effects and patients' satisfaction}

No statistically significant differences were observed between the studied groups with regard to the total number of patients experienced adverse effects over the trial. Minor gastrointestinal complaints were the most reported adverse effects among both groups with 5 patients (12.5\%) in vitamin D group and 8 patients $(22.2 \%)$ in controls. None of the studied subjects reported any serious adverse reactions, and the interventions overly were well-tolerated. The median score of patients' satisfaction with the trial 
Table 2 Changes in migraine characteristics, the use of analgesics and serum CGRP levels before and after supplementation with vitamin D or placebo in episodic migraine patients

\begin{tabular}{|c|c|c|c|c|c|c|}
\hline & \multicolumn{2}{|c|}{ Vitamin D Group $(n=38)$} & \multicolumn{2}{|c|}{ Placebo Group $(n=36)$} & \multirow{2}{*}{$\begin{array}{l}P \text { value for } \\
\text { baseline } \\
\text { comparison }\end{array}$} & \multirow{2}{*}{$\begin{array}{l}P \text { value for } \\
\text { end of trial } \\
\text { comparison }\end{array}$} \\
\hline & Baseline & After 12 Weeks & Baseline & After 12 Weeks & & \\
\hline \multicolumn{7}{|l|}{ Serum 25OHD Levels (ng/ml) } \\
\hline Mean (SD) & $27.31(12.30)$ & $39.65(13.45)$ & $33.70(11.08)$ & $33.95(12.14)$ & 0.017 & $0.062^{£}$ \\
\hline Changes from baseline & $12.34(6.34)$ & & $-0.28(5.90)$ & & & \\
\hline$P$ value for within-group comparison & $<0.001^{\#}$ & & $0.782^{\#}$ & & & \\
\hline \multicolumn{7}{|l|}{ Number of Headache Days per month } \\
\hline Mean (SD) & $8.04(3.35)$ & $5.07(2.82)$ & 7.69 (3.39) & $7.26(3.86)$ & 0.644 & $0.006^{ \pm}$ \\
\hline Adjusted mean $(95 \% \mathrm{Cl})$ & & $4.71(3.64-5.77)$ & & $6.43(5.28-7.58)$ & & $0.031^{\delta}$ \\
\hline Changes from baseline & $-2.90(3.03)$ & & $-0.14(2.91)$ & & & \\
\hline$P$ value for within-group comparison & $<0.001^{\#}$ & & $0.776^{\#}$ & & & \\
\hline \multicolumn{7}{|l|}{ Migraine Related Disability (MIDAS score) } \\
\hline & $29.75(18.54)$ & $19.38(16.43)$ & $36.87(21.55)$ & $35.36(19.45)$ & 0.117 & $<0.001^{ \pm}$ \\
\hline Adjusted mean $(95 \% \mathrm{Cl})$ & & $21.49(16.22-26.77)$ & & $31.16(25.51-36.82)$ & & $0.016^{\delta}$ \\
\hline Changes from baseline & $-10.38(12.29)$ & & $0.33(14.82)$ & & & \\
\hline$P$ value for within-group comparison & $<0.001^{\#}$ & & $0.893^{\#}$ & & & \\
\hline \multicolumn{7}{|l|}{ Serum CGRP levels (ng/L) } \\
\hline Mean (SD) & $195.27(78.45)$ & $167.961(80.112)$ & $166.05(58.32)$ & $176.71(72.22)$ & 0.063 & $0.626^{£}$ \\
\hline Adjusted mean (95\%Cl) & & $153.26(133.03-173.49)$ & & $188.35(167.15-209.54)$ & & $0.022^{\epsilon}$ \\
\hline Changes from baseline & $-27.31(59.69)$ & & $12.57(23.85)$ & & & \\
\hline$P$ value for within-group comparison & $0.006^{\#}$ & & $0.004^{\#}$ & & & \\
\hline
\end{tabular}

$P$ value $<0.05$ was considered significant

The second row of this table was previously published in [24] in a slightly different format

$B M I$ Body mass index, MIDAS Migraine disability assessment questionnaire, CGRP Calcitonin gene-related peptide

\#Paired sample t-test

${ }^{\mathrm{E}}$ Independent t-test

${ }^{\delta}$ ANCOVA test adjusted for baseline values, age, sex, and BMI change

${ }^{\epsilon}$ ANCOVA test adjusted for baseline CGRP levels, age, sex, BMI change, the years having headache, and time from last attack

Table 3 Changes in number of headache days, and migraine disability scores before and after supplementation with vitamin D or placebo in episodic migraine patients with/without aura

\begin{tabular}{|c|c|c|c|c|c|}
\hline & \multicolumn{4}{|l|}{ Study sub-groups } & \multirow[t]{3}{*}{$P$ value ${ }^{*}$} \\
\hline & \multicolumn{2}{|c|}{ Patients with migraine with aura } & \multicolumn{2}{|c|}{ Patients with migraine without aura } & \\
\hline & Vitamin D & Placebo & Vitamin D & Placebo & \\
\hline \multicolumn{6}{|c|}{ Number of Headache Days per month } \\
\hline Baseline & $10.58(3.67)^{a, b}$ & $7.75(3.86)$ & $6.81(2.43)^{a}$ & $7.67(3.29)^{b}$ & 0.008 \\
\hline After the trial & $6.00(3.45)$ & $7.50(3.32)$ & $4.63(2.40)^{\mathrm{a}}$ & $7.17(4.11)^{\mathrm{a}}$ & 0.029 \\
\hline Changes & $-4.58(3.76)^{a, b}$ & $-0.25(3.05)^{a}$ & $-2.09(2.27)$ & $-0.10(2.91)^{b}$ & 0.000 \\
\hline$P$ value ${ }^{\#}$ & 0.001 & 0.801 & $<0.001$ & 0.868 & \\
\hline \multicolumn{6}{|c|}{ Migraine Related Disability (MIDAS score) } \\
\hline Baseline & $40.00(26.38)$ & $37.90(18.53)$ & $24.81(10.80)$ & $36.53(22.75)$ & 0.057 \\
\hline After the trial & $23.08(24.42)$ & $42.90(25.15)^{\mathrm{a}}$ & $17.59(10.94)^{a . b}$ & $32.46(16.44)^{b}$ & 0.001 \\
\hline Changes & $-16.92(15.39)^{a, b}$ & $5.00(20.18)^{a}$ & $-7.22(9.25)$ & $-1.46(12.20)^{b}$ & 0.001 \\
\hline$P$ value ${ }^{\#}$ & 0.002 & 0.453 & $<0.001$ & 0.547 & \\
\hline
\end{tabular}

Data are presented as mean (standard deviation)

Alphabets represent significant differences between each variable and two other variables, calculated by Bonferroni test (post-hoc)

*One-way analysis of variance (ANOVA)

\# Paired sample t-test 


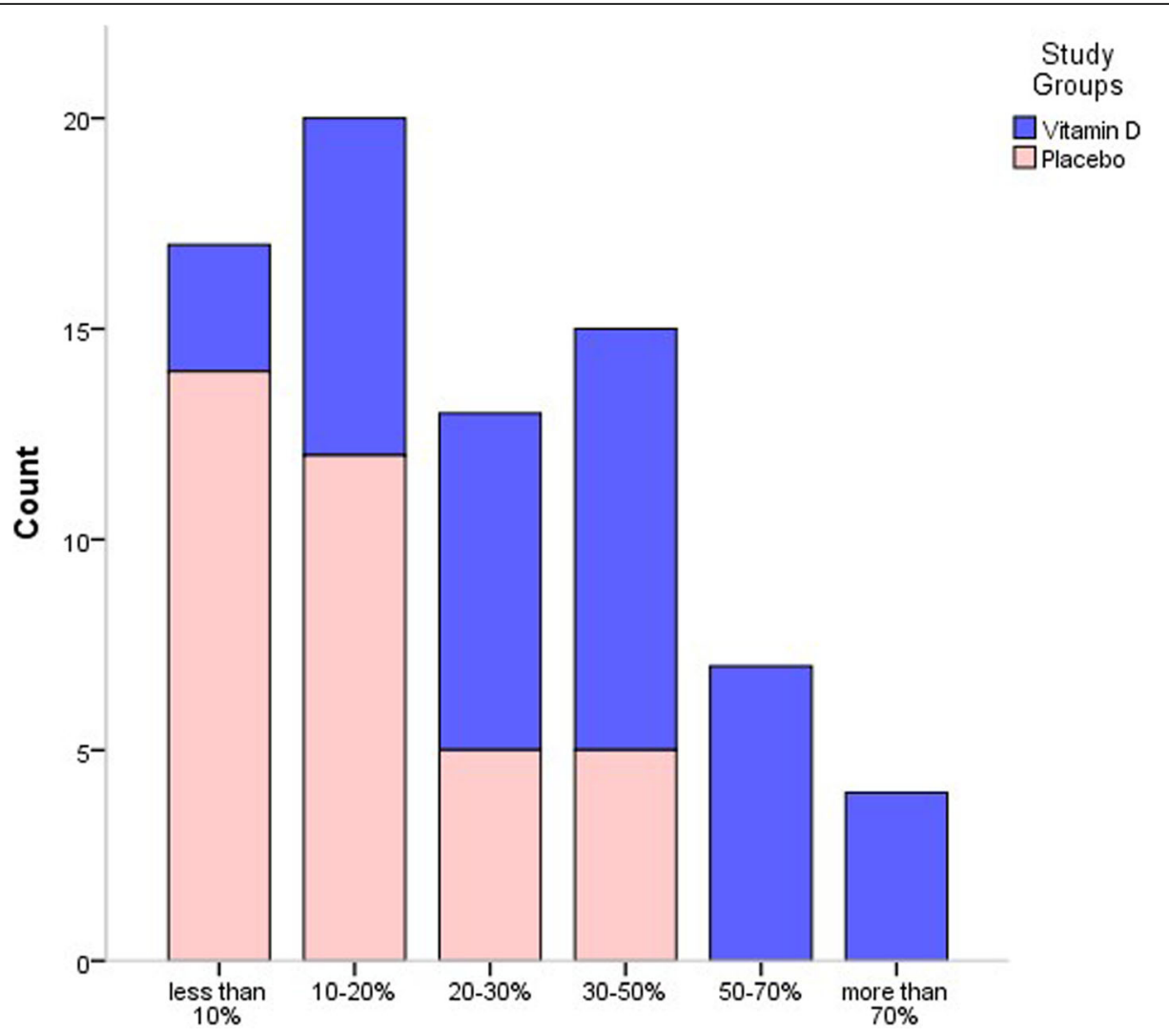

Patients Overall Perception of Headache Improvement

Fig. 3 Patients Overall Perception of Headache Improvement Following 12-week Trial. ( $P$ value $<0.001)$

was higher among vitamin $\mathrm{D}$ receiving patients (median = $3)$ than placebo groups $($ median $=2)(P$ value: 0.003$)$.

\section{Discussion}

In our previous report of present RCT, it was first demonstrated that 12 -week supplementation with vitamin D $2000 \mathrm{IU} / \mathrm{d}$ resulted in significantly lower headache days, and attacks frequency [24]. Further analysis in current study, showed that following adjustment for confounding factors (i.e. baseline disability level, sex, age, and BMI change) using ANCOVA, vitamin D is shown to significantly decrease migraine related disability in comparison with placebo group. Interestingly, these results were especially pronounced among those patients who had migraine with aura. Current study may be considered a further effort in clarifying the mechanisms through which vitamin $\mathrm{D}$ might be effective in migraine prophylaxis. In this regard, it is demonstrated that following the trial, mean interictal level of CGRP in patients who received $2000 \mathrm{IU} /$ $\mathrm{d}$ of vitamin D was significantly lower than that of placebo receiving group.

These results tie well with most of the previous studies wherein vitamin D supplementation effects were investigated in relation to pain, headache or migraine [8, 29-33].
According to the recently published review article, there have been numerous studies that reported a positive linkage between vitamin D deficiency and pain associated conditions; however, no causal relationship has been noted yet [8]. There are also clinical studies that have found vitamin D supplementation may result in pain relieving effects particularly among the subjects who had S25OHD concentration less than $30 \mathrm{nmol} / \mathrm{L}$ [29-33].

There are several likely mechanisms that may explain the protective effects of vitamin $\mathrm{D}$ on migraine characteristics and associated disability. First, according to current results, administration of this vitamin significantly reduced serum CGRP levels, the dominant mediator [25] of migraine pain. To our knowledge current study results regarding the effects of vitamin D on CGRP are novel, therefore we cannot directly compare them with previous studies.

CGRP is a vasoactive neuropeptide containing 37 amino acids that plays a role in vasodilation, modulating immune system, neuronal inflammation and transmission of pain signals. Two main isoforms of CGRP are alpha-CGRP (which is certainly found in both peripheral and central nervous system (PNS and CNS)) and beta-CGRP (which is mainly present in enteric nervous system), only differ in 

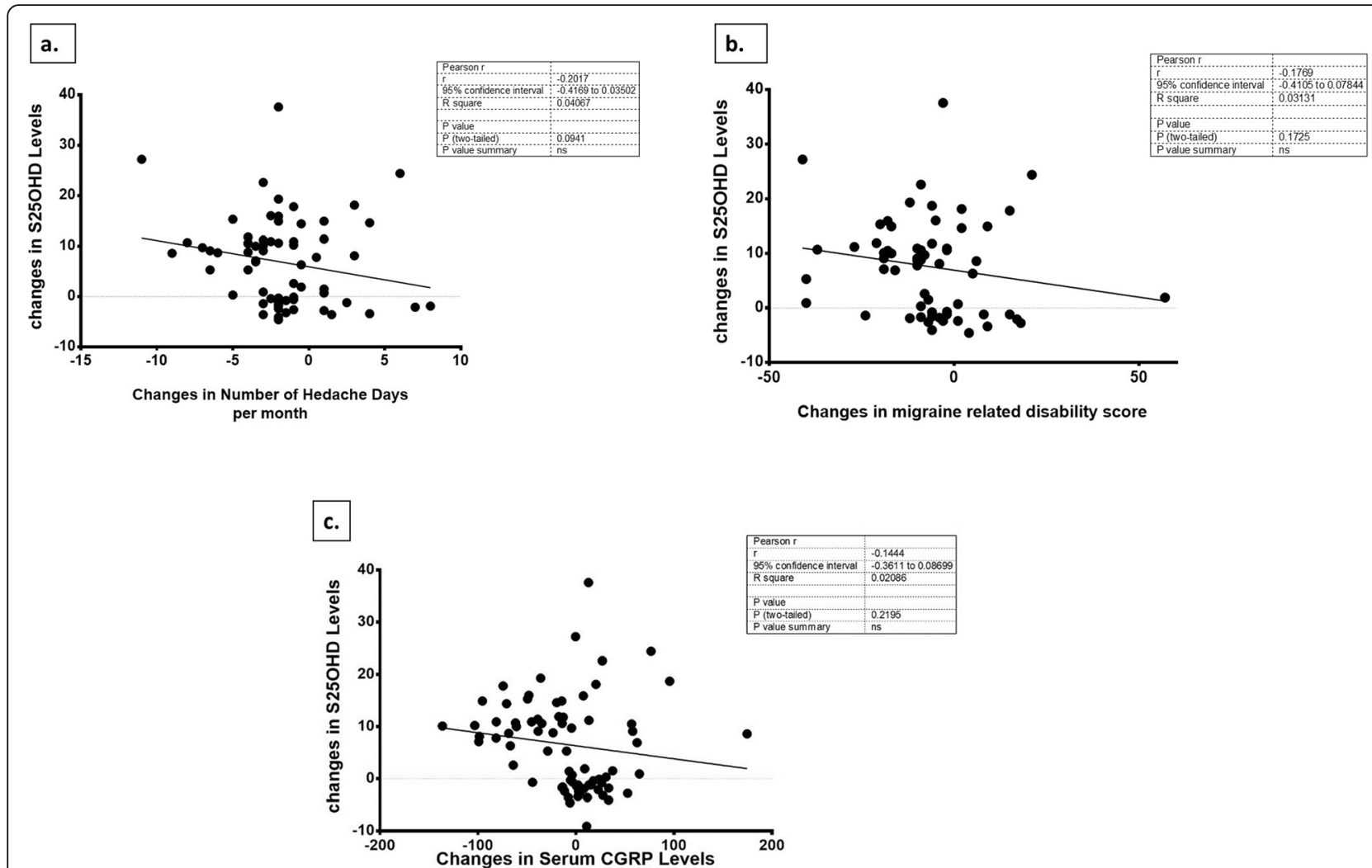

Fig. 4 a The Correlation between changes in serum 25OHD and number of headache days per month throughout 12-week trial. b The Correlation between changes in serum 25OHD and migraine related disability score (MIDAS, migraine Disability Assessment score) throughout 12-week trial. c The Correlation between changes in serum 25OHD and CGRP (calcitonin gene-related peptide) levels throughout 12-week trial

3 amino acid constituents [34]. The sensory nerve fibers that contain CGRP are particularly expressed in neuronal and vascular tissues of the cerebrum, specifically in the trigeminal ganglion. This neuropeptide could induce direct vasodilatation (independent of endothelium) through binding to the receptors (CGRP-1, CGRP-2, CGRP-3) and stimulating the release of adenyl cyclase and cyclic adenosine monophosphate (cAMP). It is thought that serotonergic system regulate CGRP secretion while nerve growth factor and NO affect its production. Additionally, CGRP is highly interacted with substance P (SP) such that both neuropeptides could usually be found in the same sites of nervous system $[25,34,35]$. As one of the most studied factors in relation to migraine, CGRP is contributed to a variety of pathophysiological mechanisms involved in pain sensitization in this type of headache. Inducing dilation in the blood vessels of dura matter, causing degranulation of mast cells and above all, playing a pivotal role in activation of trigeminovascular system which is responsible for nociceptive information conduction from the meninges to the cortex and central region of the brain, are among the suggested mechanisms [34].

The CGRP concentration is detected to be augmented in migraineurs especially during head pain periods [34, 36].
These evidences can further confirm the hypothesis of recognizing migraine as a neuro-vascular disorder and also support the potent role of CGRP and neural events that cause dilation in blood vessels in migraine pathogenesis $[34,36]$. In most of the subjects who had a history of migraine, a delayed migraine attack occurs following CGRP administration in IV form; while CGRP administration in healthy subjects did not result in pain sensitization including headache or somatic pain. Therefore, it seems that CGRP could not directly cause migraine pain and may mainly impose its effects via induction of different pathways such as stimulating the production of nitric oxide (NO), that finally could lead to neuro-inflammation and vasodilation which both might be involved in migraine pain $[25,36]$. CGRP in combination with SP, PACAP, glutamate and neuropeptide $Y$ could also induce a neurogenic inflammatory state in the CNS especially in trigeminovascular system. This inflammatory state is thought to be contributed to migraine pain sensation. Nonetheless, the activation of trigeminal pathway could also provoke the release of these factors and particularly might increase CGRP and SP secretion $[9,10,25,34,35,37-40]$.

To our knowledge this is the first investigation in migraineurs that assessed the levels of CGRP following vitamin D 
supplementation. According to available evidence, the mechanisms through which vitamin D influence CGRP synthesis and or release have not been clearly defined yet. There are a number of studies in which the effects of vitamin D or its derivatives on CGRP concentration were evaluated in animal models or cell cultures in different conditions; however, the findings have been inconclusive [41-43] due, in part, to the animal models applied or the dosage/duration of vitamin $\mathrm{D}$ administration.

In the present research, it was found that patients in the intervention group had significantly lower S25OHD levels, and on the other hand, a slightly higher serum CGRP levels at baseline; however, supplementation with the vitamin resulted in significant increment of S25OHD and reduction of CGRP, though we did not find any dose-response correlation throughout the trial between either of headache related parameters (e.g. frequency of headache days, MIDAS score or serum CGRP levels) and S25OHD. Therefore, it is likely that the protective effects of vitamin $D$ in ameliorating migraine related features and CGRP levels reduction may be attributed to its anti-nociceptive properties and some indirect pathways. It is worth noting that NO, might elevate CGRP and SP synthesis/secretion release that ultimately may result in triggering nociceptive neurons and inflammation specially in trigeminovascular system [25, 34, 35, 39, 44]. On the flip side, decreased vitamin D concentration has been linked to elevated levels of NO. The protective efficacy of this vitamin against endothelial dysfunction as well as cardiovascular system could also be explained through this inverse relationship between $\mathrm{NO}$ and S5OHD levels [45]. Further, glutathione (GSH) presence in astrocytes may be associated with nitrogen and oxygen reactive species eliminating procedure (such as $\mathrm{NO}$ ) $[46,47]$. Since vitamin D active form $(1,25(\mathrm{OH}) 2 \mathrm{D} 3)$ may be involved in GSH metabolism, it can be speculated that vitamin D may also be involved in NO metabolism pathway [8].

\section{Study limitations}

The strengths of this study are the prospective- double blind, placebo- controlled design, using a safe daily dosage of vitamin $\mathrm{D}$ as a somewhat inexpensive and available overthe-counter supplement, exploring CGRP levels as the dominant indicator of migraine pathogenesis, enrolling the study participant following examinations by expert headache-specialist neurologists, and confirming episodic migraine diagnosis based on the most recent ICHD criteria (ICHDIII). In spite of these strengths, there were a number of limitations. First of all, due to ethical considerations and according to migraine trials guidelines [27], it was not possible to discontinue the patients concomitant pharmacological therapy (including prophylactic or acute drugs). However, patients were recommended not to change their medications during the study. Also those subjects who received the medications that are supposed to interact with vitamin D were excluded from the trial. Second, although we tried to consider the effects of some confounding factors on the influences of vitamin D supplementation on headache outcomes through the ANCOVA, there might still be some other factors that could have been taken into account. Third, although both cases and controls were selected from the same population with similar characteristics including demographic, anthropometric and socioeconomic status, there could still be a chance of bias. Further, the current study was not powered enough to conduct a subgroup analysis between those with vitamin D deficiency/insufficiency. Thus, in future trials, subjects could be categorized according to S25OHD status so that appropriate dosage adjustment of vitamin $\mathrm{D}$ can be made. Additionally, investigating the efficacy of vitamin $\mathrm{D}$ on subgroups of migraine patients can be considered in the future trials including those with menstrual related migraine, chronic migraine or migraine with/without aura. Also, the effect of vitamin $\mathrm{D}$ receptor gene polymorphism should be clarified when studying the potential role of vitamin $\mathrm{D}$ supplementation in improvement of migraine characteristics and disability level.

\section{Conclusion}

According to the current findings, vitamin D supplementation may potentially improve migraine headache, which has been established as the leading cause of disability in $<50$ years, probably through attenuating CGRP levels. Therefore, daily administration of vitamin D 2000 IU as an adjuvant therapy could be considered a welltolerated and effective agent in improvement of migraine characteristics and disability level particularly among those patients who have migraine with aura. This could provide a new insight into anti-nociceptive effects of vitamin D. However, to confirm current promising findings, large sample size RCTs with longer duration of follow-up are required especially in patients with chronic migraine. Also, well-designed animal and/or clinical studies addressing the mechanisms through which vitamin D may influence migraine pathogenesis, are of crucial importance.

\footnotetext{
Abbreviations

ANCOVA: Analysis of covariance; cAMP: Cyclic adenosine monophosphate; CGRP: Calcitonin gene-related peptide; Cl: Confidence interval;

CLIA: Chemiluminescence immunoassays; CSD: Cortical spreading depression; ELIZA: Enzyme linked immunosorbent assay; EM: Episodic migraine;

GBD: Global burden of disease; GSH: Glutathione; ICHDIII

criteria: International Classification of Headache Disorders, 3rd edition;

IL: Interleukin; iNOS: Inducible nitric oxide synthase; MIDAS: Migraine Disability Assessment Questionnaire; NO: Nitric oxide; PACAP: Pituitary adenylate cyclase-activating peptide; PNS and CNS: Peripheral and central nervous system; RCT: Randomized clinical trial; SD: Standard deviation; serum 25OHD: S25OHD; SP: Substance P; TNF-a: Tumor necrosis factor- a
} 


\section{Acknowledgments}

The authors would like to thank the participants of the study for their cooperation.

\section{Authors' contributions}

ZGh, MT, and MM: Conceived and designed the work that led to the submission, acquired data, and played an important role in interpreting the results. ZGh, MT, MM, PR, SH, RRM, ZSA, MDj, MZ, SRJ, and SSh: Acquired data. ZGh, MT, MM, and AF: Played an important role in interpreting the results. ZGh, MT, and MM: Drafted the manuscript. ZGh, MT, MM, PR, AF, SH RRM, ZSA, MDj, MZ, SRJ, and SSh approved the final version and agreed to be accountable for all aspects of the work in ensuring that questions related to the accuracy or integrity of any part of the work are appropriately investigated and resolved. MM and MT are equally responsible as corresponding authors.

\section{Funding}

This research has been supported by Tehran University of Medical Sciences \& health Services grant 96-04-161-37647 and 96-04-54-36870.

\section{Availability of data and materials}

The datasets of the current study are available from the corresponding authors upon reasonable request.

\section{Ethics approval and consent to participate}

The study protocol was in accordance with Helsinki Declaration, and was approved by the ethics committee of Tehran university of medical sciences (ethics code: IR.TUMS.VCR.REC.1397.170, approve date: 2018-04-08). Also, at the beginning of the study, after describing the aims and possible risks or benefits of the trial, all participants provided written informed consent. The trial is registered in the Iranian registry of clinical trials (IRCT) with IRCT code: IRCT20151128025267N6. This research has been supported by Tehran University of Medical Sciences grant number 96-04-161-37647 and 96-04-5436870

\section{Consent for publication}

All authors consented.

\section{Competing interests}

The authors declare that they have no competing interests.

\section{Author details}

'Department of Cellular and Molecular Nutrition, School of Nutritional Sciences and Dietetics, Tehran University of Medical Sciences, Tehran, Iran. ${ }^{2}$ Student Research Committee, Department and Faculty of Nutrition Sciences and Food Technology, Shahid Beheshti University of Medical Sciences, Tehran, Iran. ${ }^{3}$ Department of Epidemiology and Biostatistics, School of Public Health, Tehran University of Medical Sciences, Tehran, Iran. ${ }^{4}$ Headache Department, Iranian Center of Neurological Research, Neuroscience Institute, Tehran University of Medical Sciences, Tehran, Iran. ${ }^{5}$ Department of Nutrition, Faculty of Medical Sciences, Science and Research Branch, Islamic Azad University, Tehran, Iran. ${ }^{6}$ Department of Nutrition, School of Health, Iran University of Medical Sciences, Tehran, Iran. ${ }^{7}$ Department of Clinical Nutrition and Dietetics, Faculty of Nutrition and Food Technology, Shahid Beheshti University of Medical Sciences, Tehran, Iran. ${ }^{8}$ Nutrition and Endocrine Research Center, Research Institute for Endocrine Sciences, Department of Clinical Nutrition and Dietetics, Shahid Beheshti University of Medical Sciences, Tehran, Iran. ${ }^{9}$ Pediatric Gastroenterology and Hepatology Research Center, Children's Medical Center, Tehran University of Medical Sciences, Tehran, Iran. ${ }^{10}$ Dietitians and Nutrition Experts Team (DiNET), Universal Scientific Education and Research Network (USERN), Tehran, Iran. ${ }^{11}$ Headache Department, Neurology Ward, Sina University Hospital, School of Medicine, Tehran University of Medical Sciences, Tehran, Iran.

Received: 6 January 2020 Accepted: 13 February 2020

\section{Published online: 24 February 2020}

\section{References}

1. Steiner TJ, Stovner LJ, Vos T, Jensen R, Katsarava Z (2018) Migraine is first cause of disability in under 50s: will health politicians now take notice? J Headache Pain 19(1):17
2. Stovner L, Hagen K, Jensen R, Katsarava Z, Lipton R, Scher A et al (2007) The global burden of headache: a documentation of headache prevalence and disability worldwide. Cephalalgia 27(3):193-210

3. Steiner TJ, Stovner LJ, Vos T (2016) GBD 2015: migraine is the third cause of disability in under 50 s

4. Vetvik KG, MacGregor EA (2017) Sex differences in the epidemiology, clinical features, and pathophysiology of migraine. Lancet Neurol 16(1):76-87

5. Headache Classification Committee of the International Headache Society (IHS) (2018) The international classification of headache disorders, 3rd edition. Cephalalgia 38(1):1-211

6. Katsarava Z, Schneeweiss S, Kurth T, Kroener U, Fritsche G, Eikermann A et al (2004) Incidence and predictors for chronicity of headache in patients with episodic migraine. Neurology 62(5):788-790

7. Buse DC, Manack AN, Fanning KM, Serrano D, Reed ML, Turkel CC et al (2012) Chronic migraine prevalence, disability, and sociodemographic factors: results from the American migraine prevalence and prevention study. Headache 52(10):1456-1470

8. Ghorbani Z, Togha M, Rafiee P, Ahmadi ZS, Rasekh Magham R, Haghighi S et al (2019) Vitamin D in migraine headache: a comprehensive review on literature. Neurol Sci 40(12):2459-2477

9. Dodick DW (2018) A phase-by-phase review of migraine pathophysiology. Headache 58(Suppl 1):4-16

10. Pietrobon D (2005) Migraine: new molecular mechanisms. Neuroscientist 11(4):373-386

11. Togha M, Razeghi Jahromi S, Ghorbani Z, Ghaemi A, Rafiee P (2019) An investigation of oxidant/antioxidant balance in patients with migraine: a case-control study. BMC Neurol 19(1):323

12. Adams J, Barbery G, Lui CW (2013) Complementary and alternative medicine use for headache and migraine: a critical review of the literature. Headache 53(3):459-473

13. Silberstein SD, Holland S, Freitag F, Dodick DW, Argoff C, Ashman E (2012) Evidence-based guideline update: pharmacologic treatment for episodic migraine prevention in adults: report of the quality standards subcommittee of the American Academy of Neurology and the American Headache Society. Neurology 78(17):1337-1345

14. Hepp Z, Bloudek LM, Varon SF (2014) Systematic review of migraine prophylaxis adherence and persistence. J Manag Care Pharm 20(1):22-33

15. Blumenfeld AM, Bloudek LM, Becker WJ, Buse DC, Varon SF, Maglinte GA et al (2013) Patterns of use and reasons for discontinuation of prophylactic medications for episodic migraine and chronic migraine: results from the second international burden of migraine study (IBMS-II). Headache 53(4):644-655

16. Nattagh-Eshtivani E, Sani MA, Dahri M, Ghalichi F, Ghavami A, Arjang P et al (2018) The role of nutrients in the pathogenesis and treatment of migraine headaches: review. Biomed Pharmacother 102:317-325

17. Wells RE, Beuthin J, Granetzke L (2019) Complementary and integrative medicine for episodic migraine: an update of evidence from the last 3 years. Curr Pain Headache Rep 23(2):10

18. Gazerani P, Fuglsang R, Pedersen JG, Sorensen J, Kjeldsen JL, Yassin $\mathrm{H}$ et al (2019) A randomized, double-blinded, placebo-controlled, parallel trial of vitamin D3 supplementation in adult patients with migraine. Curr Med Res Opin 35(4):715-723

19. Buettner C, Nir RR, Bertisch SM, Bernstein C, Schain A, Mittleman MA et al (2015) Simvastatin and vitamin D for migraine prevention: a randomized, controlled trial. Ann Neurol 78(6):970-981

20. Yilmaz R, Salli A, Cingoz HT, Kucuksen S, Ugurlu H (2016) Efficacy of vitamin D replacement therapy on patients with chronic nonspecific widespread musculoskeletal pain with vitamin D deficiency. Int J Rheum Dis 19(12):1255-1262

21. Mottaghi T, Askari G, Khorvash F, Maracy MR (2015) Effect of vitamin D supplementation on symptoms and $\mathrm{C}$-reactive protein in migraine patients. J Res Med Sci 20(5):477-482

22. Knutsen KV, Madar AA, Brekke M, Meyer HE, Natvig B, Mdala I et al (2014) Effect of vitamin D on musculoskeletal pain and headache: a randomized, double-blind, placebo-controlled trial among adult ethnic minorities in Norway. Pain 155(12):2591-2598

23. Cayir A, Turan MI, Tan H (2014) Effect of vitamin D therapy in addition to amitriptyline on migraine attacks in pediatric patients. Braz J Med Biol Res 47(4):349-354

24. Ghorbani Z, Togha M, Rafiee P, Ahmadi ZS, Rasekh Magham R, Djalali M et al (2020) Vitamin D3 might improve headache characteristics and protect against inflammation in migraine: a randomized clinical trial. Neurol Sci. https://doi.org/10.1007/s10072-019-04220-8. [Epub ahead of print] 
25. Edvinsson L (2017) The trigeminovascular pathway: role of CGRP and CGRP receptors in migraine. Headache 57(Suppl 2):47-55

26. Razeghi Jahromi S, Abolhasani M, Ghorbani Z, Sadre-Jahani S, Alizadeh Z, Talebpour M et al (2018) Bariatric surgery promising in migraine control: a controlled trial on weight loss and its effect on migraine headache. Obes Surg 28(1):87-96

27. Tfelt-Hansen P, Pascual J, Ramadan N, Dahlof C, D'Amico D, Diener HC et al (2012) Guidelines for controlled trials of drugs in migraine: third edition. A guide for investigators. Cephalalgia 32(1):6-38

28. Zandifar A, Asgari F, Haghdoost F, Masjedi SS, Manouchehri N, Banihashemi M et al (2014) Reliability and validity of the migraine disability assessment scale among migraine and tension type headache in Iranian patients. Biomed Res Int 2014:7

29. Helde-Frankling M, Bjorkhem-Bergman L (2017) Vitamin D in pain management. Int J Mol Sci 18(10):2170

30. Yong WC, Sanguankeo A, Upala S (2017) Effect of vitamin D supplementation in chronic widespread pain: a systematic review and meta-analysis. Clin Rheumatol 36(12):2825-2833

31. de Oliveira DL, Hirotsu C, Tufik S, Andersen ML (2017) The interfaces between vitamin D, sleep and pain. J Endocrinol 234(1):R23-R36

32. Zadro J, Shirley D, Ferreira M, Carvalho-Silva AP, Lamb SE, Cooper $C$ et al (2017) Mapping the association between vitamin D and low back pain: a systematic review and meta-analysis of observational studies. Pain Physician 20(7):611-640

33. Wu Z, Malihi Z, Stewart AW, Lawes CM, Scragg R (2016) Effect of vitamin D supplementation on pain: a systematic review and meta-analysis. Pain Physician 19(7):415-427

34. Malhotra R (2016) Understanding migraine: potential role of neurogenic inflammation. Ann Indian Acad Neurol 19(2):175-182

35. Melinda L, Janos T, Ferenc F, Jozsef T, Lars E, Laszlo V (2017) Migraine, neurogenic inflammation, drug development - pharmacochemical aspects. Curr Med Chem 24(33):3649-3665

36. lyengar S, Johnson KW, Ossipov MH, Aurora SK (2019) CGRP and the trigeminal system in migraine. Headache 59(5):659-681

37. Ceylan M, Bayraktutan OF, Becel S, Atis O, Yalcin A, Kotan D (2016) Serum levels of pentraxin-3 and other inflammatory biomarkers in migraine: association with migraine characteristics. Cephalalgia 36(6):518-525

38. Palm-Meinders $I H$, Koppen $H$, Terwindt $G M$, Launer $\sqcup$, Konishi J, Moonen JM et al (2012) Structural brain changes in migraine. JAMA 308(18):1889-1897

39. Ramachandran R (2018) Neurogenic inflammation and its role in migraine. Semin Immunopathol 40(3):301-314

40. Sadeghi O, Nasiri M, Maghsoudi Z, Pahlavani N, Rezaie M, Askari G (2015) Effects of pyridoxine supplementation on severity, frequency and duration of migraine attacks in migraine patients with aura: a double-blind randomized clinical trial study in Iran. Iran J Neurol 14(2):74-80

41. Riaz S, Malcangio M, Miller M, Tomlinson DR (1999) A vitamin D(3) derivative (CB1093) induces nerve growth factor and prevents neurotrophic deficits in streptozotocin-diabetic rats. Diabetologia 42(11):1308-1313

42. Cote GJ, Rogers DG, Huang ESC, Gagel RF (1987) The effect of 1,25dihydroxyvitamin D3 treatment on calcitonin and calcitonin gene-related peptide mRNA levels in cultured human thyroid C-cells. Biochem Biophys Res Commun 149(1):239-243

43. Tague SE, Smith PG (2011) Vitamin D receptor and enzyme expression in dorsal root ganglia of adult female rats: modulation by ovarian hormones. J Chem Neuroanat 41(1):1-12

44. Olesen J (2008) The role of nitric oxide (NO) in migraine, tension-type headache and cluster headache. Pharmacol Ther 120(2):157-171

45. Al-Daghri NM, Bukhari I, Yakout SM, Sabico S, Khattak MNK, Aziz I et al (2018) Associations of serum nitric oxide with vitamin $D$ and other metabolic factors in apparently healthy adolescents. Biomed Res Int 2018:1489132

46. Shipton EA, Shipton EE (2015) Vitamin D and pain: vitamin D and its role in the aetiology and maintenance of chronic pain states and associated comorbidities. Pain Res Treat 2015:904967

47. Garcion E, Sindji L, Leblondel G, Brachet P, Darcy F (1999) 1,25dihydroxyvitamin $\mathrm{D} 3$ regulates the synthesis of gamma-glutamyl transpeptidase and glutathione levels in rat primary astrocytes. J Neurochem 73(2):859-866

\section{Publisher's Note}

Springer Nature remains neutral with regard to jurisdictional claims in published maps and institutional affiliations.

\section{Ready to submit your research? Choose BMC and benefit from:}

- fast, convenient online submission

- thorough peer review by experienced researchers in your field

- rapid publication on acceptance

- support for research data, including large and complex data types

- gold Open Access which fosters wider collaboration and increased citations

- maximum visibility for your research: over $100 \mathrm{M}$ website views per year

At BMC, research is always in progress.

Learn more biomedcentral.com/submissions 